\title{
Efficient Active Monocular Fixation using the Log-polar Sensor
}

\author{
Albert Yeung ${ }^{1}$ Nick Barnes ${ }^{1,2}$ \\ ${ }^{1}$ Department of Computer Science \\ and Software Engineering, \\ University of Melbourne, Victoria, 3010, \\ AUSTRALIA. \\ 2 National ICT Australia, Locked Bag 8001, \\ Canberra, ACT 2601, AUSTRALIA. \\ Nick.Barnesenicta.com.au
}

\begin{abstract}
This paper applies a sensor which has been developed based on a model of primate vision to corner fixation. Fixation is critical for the log-polar sensor as many of the sensor's advantages are demonstrated only under fixation. This paper presents a fast corner fixation algorithm using the log-Hough transform to find the dominant centre-most corner and aligning the optical axis with the corner. The algorithm has been implemented in a real-time closed-loop control system on a pan-tilt platform, This paper demonstrates an important benefit of the log-polar sensor with vote-based algorithms, an emphasis of foveal pixels. This removes the need for searching explicitly through all detected corner positions in order to locate the most central. In addition, since the sensor has a large field of view for a given number of pixels compared to space-invariant cameras, it shows increased computational performance, while still supporting accurate fixation.
\end{abstract}

Keywords: Vision, Robotics, biologically inspired robots, biologically inspired sensing, fixation, log-polar sensor.

\section{INTRODUCTION}

A deeper understanding of human anatomy and biology is contributing greatly to enhancing health and quality of human life through avenues such as medicine and education. While direct study of human anatomy and behaviour yields many insights, there are limitations when studying biological creatures as systems due to their extreme complexity. Robotic simulation of biological entities can help here by testing whether models of perception and action can actually work in practice, or suggesting possible ways a biological entity may process information and act. On the other hand biological creatures are able to operate within their environments in a highly effective manner, far more so than the current generation of robots. Examination of biological models of perception and action can lead to more effective algorithms and systems for performing robotic tasks. These are the complementary aims of the field of bio-robotics.

The log-polar sensor was developed based on a model of primate vision [20]. Its incorporation into robotic perceptuomotor loops can help facilitate the study of biological perception and action. The log-polar sensor has a high resolution at the fovea, which reduces logarithmically towards the periphery. The sensing elements are aligned in a polar layout. Theoretical and robotic study of the sensor has lead to the discovery of various advantages for perception and action over traditional Cartesian cameras. A foundational theoretical paper by Weiman and Chaikin [27] proved that the exponential mapping is conformal (angles are preserved locally), and that expansion and rotation about the camera centre are mapped simply to addition in log-polar coordinates. Massone, Sandini and Tagliasco [15] demonstrated the advantages of the sensor for $2 \mathrm{D}$ recognition. Questa and Sandini [19] and Tisarelli and Sandini [23] showed that time-to-contact calculations are simplified, providing benefits in docking and obstacle avoidance. The natural foveation of the sensor naturally leads to a wider field of view with lesser computation requirements to maintain high resolution at region of interest. Bernardino and SantosVictor [4] demonstrated the advantages of distance weighting from the centre of the scene for focus of attention. Barnes and Sandini [3] showed that egomotion recovery under fixation is simplified on a log-polar sensor, benefitting docking behaviours. Weiman [26] showed the mapping of circles, straight-lines, and parabolas is unified under a $\log$ polar sensor and that the Hough transform and log-polar coordinate systems can be unified.

With these well-known advantages, this paper explores the advantages of the log-polar sensor for corner fixation. Foveation shows the greatest benefit when the sensor is centred on the region of interest in the environment, so algorithms for fixation are critical to the application of the log-polar sensor. At the same time the sensor layout is highly beneficial for fixation as it allows high-precision fixation at the centre, while maintaining a wide field of view, with a reduced total pixel count leading to faster computation. It is not as important to have as precise information about object position when the object is towards the periphery, as here the main task is to shift the object towards the fovea.

Fixation is a specialisation of visual tracking, where gaze direction is maintained on the same target point over time [1]. In general, fixation aims to maintain objects of interest at the centre of the camera - the optical axis. This maximises the distance the object can move between frames before it is lost from the field of view. Fixation is part of the active vision paradigm [2], where the observer is manipulated to obtain extra information about the environment. This ability to fixate simplifies many higher-level vision problems, such 
as docking [3]. Tracking itself is a fundamental operation in robotic vision to facilitate control (e.g., [12], [5]). It also facilitates image understanding by recovering motion [22] and through recognition of object [25] and shape [6]. Many solutions exist for space invariant cameras. However, these often utilise a sub-sampling window to reduce the pixel count [14] and so image processing time. In any case, for space invariant cameras it is always necessary to trade-off fixation precision and field of view to facilitate real-time performance.

There have been a number of fixation algorithms that have exploited the advantages of foveation of the logpolar camera. Oshiro et al. [18] present a binocular fixation algorithm exploiting the zero disparity field, which is not relevant to work on monocular fixation. In terms of algorithms that are directly relevant to monocular fixation, there have been template-based algorithms that are suitable for general fixation for the log-polar domain. Bernardino and Santos-Victor [4] formulated a template-based binocular fixation algorithm using the visual servoing framework. Ahrns and Neumann [1] proposed a view-based fixation control algorithm using monocular vision, which involves comparison against a reference view to obtain a similarity measure. These template-based algorithms are general in nature, but this generality comes at a computational cost.

However, when a corner is visible (e.g., manufactured objects) corner-based methods should be exploited for the speed increases that are possible through specialised algorithms. Corner-based fixation also can be used to establish a precise correspondence between a fixated target and a corresponding object model, and thus facilitate knowledgebased interaction. However, Cartesian-based corner tracking methods are not directly applicable to log-polar domain, thus a new approach is needed to combine the data reduction advantages of the log-polar sensor with gains from specialised corner fixation. In this research, we focus on corner fixation because corners are commonly encountered indoors and in built environments.

Corner detection is a well-researched field, with corners widely used as point features for visual reconstruction [9] and Simultaneous Localisation and Mapping (SLAM) [21]. A basic definition of a corner can be based on curvature or intensity discontinuity of edges. One of the most commonly used is the Harris corner algorithm [10]. For corner detection, an evaluation of classical algorithms is available in [7], and an empirical comparison of various types of feature detectors for reconstruction tasks can be found in [17]. The log-polar sensor is conformal so standard corner algorithms can be used with the sensor. However, there are two reasons why local point corner detection is not appropriate for this work: instability; and the singularity of the log-polar sensor. Corner detectors typically report fairly high false positive rates [17]. This is acceptable in a reconstruction or SLAM situation as matching algorithms deal with false positives. However, false positives may be highly distracting for fixation. Even for non-false positive points, point corners are not stable over different scales. For fixation, either the robot or target will be in motion. If the

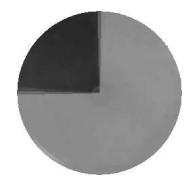

(a)

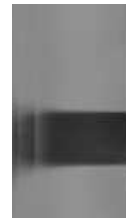

(b)

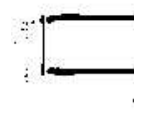

(c)
Fig. 1. A corner the centre: (a) Cartesian remapped image, (b) log-polar image, (c) log-polar edge map.

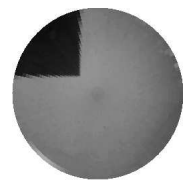

(a)

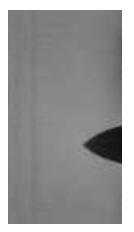

(b)

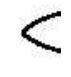

(c)
Fig. 2. A corner at an arbitrary position: (a) Cartesian remapped image, (b) log-polar image, (c) log-polar edge map.

relative motion changes the distance between the target and robot, scale will be effected. If distance increases, small corners may reduce below sensor resolution, as distance increases, what appeared as a corner at one scale may not be a corner at higher resolution. Similarly with changes in resolution across a space-variant sensor. There is work on making corners robust under scale [16], but this degrades under large scale changes. Further, the log-polar sensor has a singularity at the centre of projection. Thus, point corners will disappear as soon as they are correctly fixated. In this paper, the approach emphasises structural support of corners by defining a corner as the intersection of two straight lines to support stable fixation. This definition gives rise to a simple and fast detection algorithm using the Hough transform.

In this paper, we present a novel algorithm to detect, track and fixate corners in log-polar space. A space-variant camera can capture the same field of view as a conventional camera at lower pixel count, eliminating the need for windowing. A log-polar camera achieves this by having the maximum spatial resolution at centre (fovea) and logarithmically reducing towards the periphery. Although this does not support tracking at high resolution across the sensor, it supports high-resolution fixation. The contributions of this paper are two-fold: a demonstration of the advantages of using the log-polar camera for fixation; and, a highly effective algorithm for monocular fixation. Specifically, the advantages demonstrated are: increased field-of-view while maintaining foveal resolution; and, an emphasis on foveal pixels for vote-based algorithms. This emphasis aligns with the goal of fixation, which is to keep the corner at the 


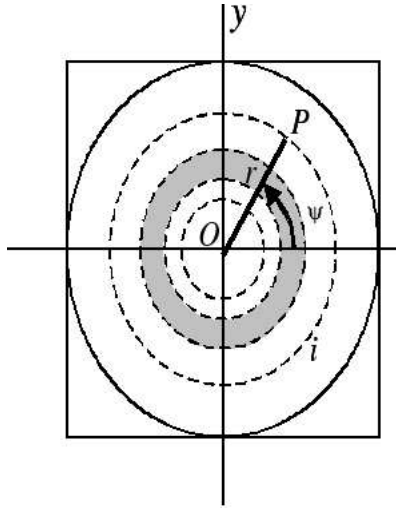

(a)

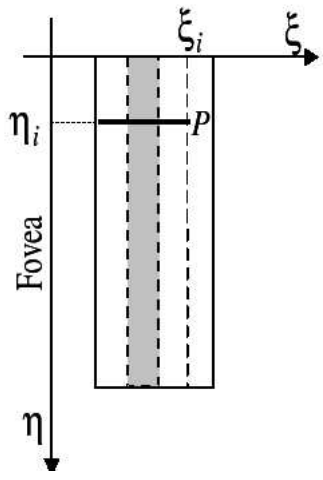

(b)
Fig. 3. An annulus in the image plane (a) maps to a vertical strip in the log-polar plane (b).

image centre. This emphasis removes the need for searching explicitly through all detected corner positions in order to locate the most central. A closed-loop control system based on the proposed fixation algorithm has been implemented and experimentally verified to be stable and sufficient for real-time performance.

\section{BACKGROUND - THE LOG-POLAR MAPPING}

The log-polar sensor imitates the mapping from the retina to visual cortex of primates [20]. In the retinal plane (Figure 3(a), a point can be represented by Cartesian coordinates $(x, y)$ or polar coordinates $(r, \psi)$ :

$$
\begin{gathered}
x=r \cos \psi, \\
y=r \sin \psi .
\end{gathered}
$$

The mapping between a polar plane $(r, \psi)$ (retinal plane) and a Cartesian plane $(\xi, \eta)$ (log-polar cortical plane, Figure 3 (b), can be written as:

$$
\begin{array}{r}
\xi=\log _{a} \frac{r}{r_{0}}, \\
\eta=q \psi,
\end{array}
$$

where $r_{0}$ is the radius of the inner-most circle and $\frac{1}{q}$ is the minimum angular resolution of the log-polar layout. A CMOS implementation of the log-polar sensor has been realised in [19] and is used in this research.

\section{ARCHITECTURE}

Figure 4 illustrates the system architecture. The control hardware consists of Giotto log-polar camera as input and a pan-tilt motor control board as output running a PID controller. The tracker software module connects these two pieces of hardware and implements the proposed corner fixation algorithm. The software architecture of the tracker is shown in Figure 5.

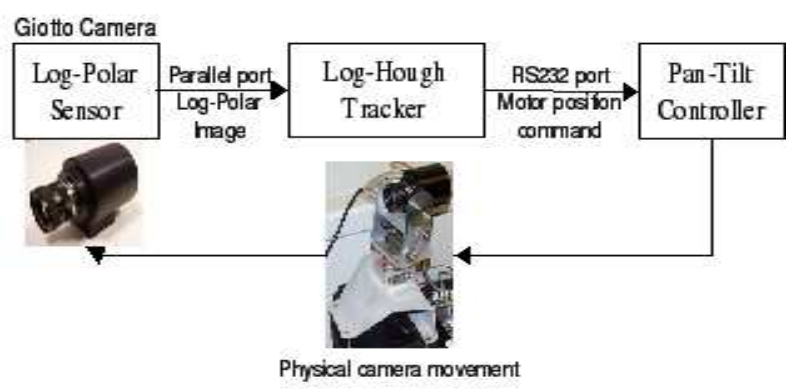

Fig. 4. Control structure of the corner tracker.

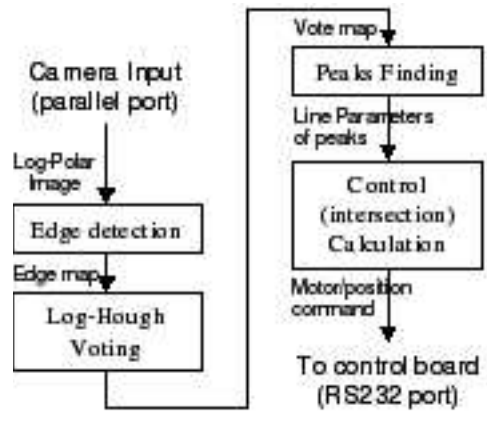

Fig. 5. Log-Hough corner tracking algorithm.

\section{THEORY}

The Hough transform [11] finds straight-lines from edge pixels in a Cartesian image. However the log-polar sensor transforms straight-lines, so the Cartesian Hough transform is not directly applicable. The log-Hough transform [26] is adapted for use in this system.

\section{A. The log-Hough transform}

Weiman's derivation [26] of the log-Hough transform begins with the following straight-line equation in polar coordinates:

$$
r=\frac{\rho}{\sin (\psi-\phi)}, 0<\psi-\phi<\pi,
$$

where $\phi$ is the slope of the line (see Figure 6). The log-polar transform of Equations (2) (3), and (3) are then applied:

$$
\xi=\log _{a} \rho-\log _{a}(\sin (\eta-\psi)) .
$$

To make use of log-Hough transform on real cameras, we must restrict the ranges to match the finite discrete representation of digital imagery. In this section, we present a full derivation of log-Hough voting equation for finite digital images. Also, Duda and Hart's [8] coordinate system should be used for consistency with standard notation in this field, as shown in Figure 6, and Equation (6). We use $(r, \theta)$ to represent polar coordinates. Hence, the standard Hough line parameterisation can be written:

$$
\rho=x \cos \theta+y \sin \theta
$$




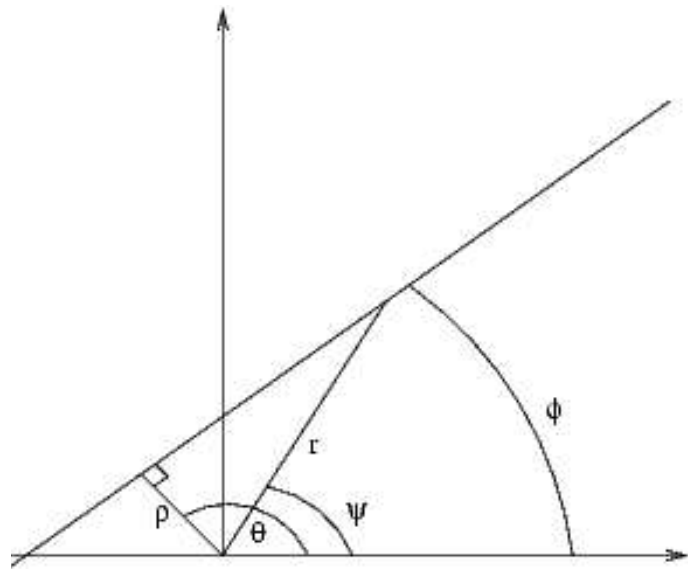

Fig. 6. Cartesian polar coordinate system.

Now, we can use $\theta$ to define the line equation in polar coordinates instead of $\phi$ :

$$
r=\frac{\rho}{\cos (\theta-\psi)}, 0<\theta-\psi<\pi, r_{0} \leq r \leq r_{\max }
$$

where $\theta$ is the angle, from $\mathrm{x}$-axis, of the perpendicular line passing through the origin. The Hough voting process increments an accumulator of all possible line parameters for a given candidate pixel. The voting equation is:

$$
\rho=r \cos (\theta-\psi), 0<\theta-\psi<\pi, r_{0} \leq r \leq r_{\max } .
$$

Converting into log polar coordinates:

$$
\rho=r_{0} a^{\xi} \cos (\eta-\psi), 0<\eta-\psi<\pi, \xi_{0} \leq \xi \leq \xi_{\max }
$$

\section{B. Intersection of two lines}

The output of the log-Hough transform is a list of recognised lines defined by two parameters $\rho_{i}$ and $\theta_{i}$ :

$$
\begin{aligned}
r & =\frac{\rho_{1}}{\cos \left(\theta_{1}-\psi\right)}, \\
r & =\frac{\rho_{2}}{\cos \left(\theta_{2}-\psi\right)} .
\end{aligned}
$$

The corner for any line pair lies at their intersection. For non-parallel lines $\left(\theta_{1} \neq \theta_{2}\right)$, an intersection can be obtained in polar coordinates from the standard equations:

$$
\begin{gathered}
r=\frac{\sqrt{\rho_{1}^{2}+\rho_{2}^{2}-\rho_{1} \rho_{2} \cos \left(\theta_{1}-\theta_{2}\right)}}{\sin \left(\theta_{1}-\theta_{2}\right)}, \\
\psi=\cos ^{-1}\left[\frac{\rho_{2} \sin \theta_{1}-\rho_{1} \sin \theta_{2}}{\sqrt{\rho_{1}^{2}+\rho_{2}^{2}-\rho_{1} \rho_{2} \cos \left(\theta_{1}-\theta_{2}\right)}}\right] .
\end{gathered}
$$

However, Equation (12) only gives the magnitude of $\psi$, the sign requires substitution into the line Equations (11) to check for consistency and obtain $r$.

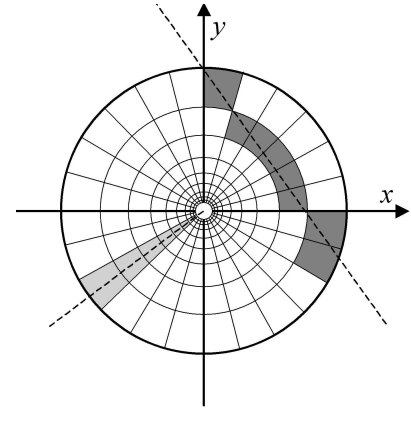

(a)

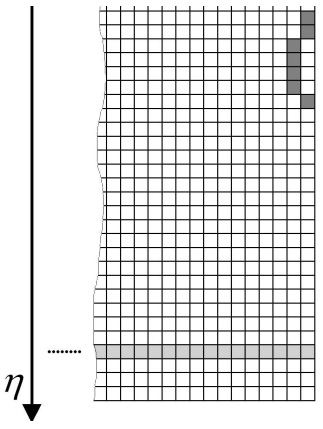

(b)
Fig. 7. Illustration of foveal voting bias. Note that the high-resolution fovea is partially shown: (a) Cartesian image domain, and (b) Log-polar image domain.

\section{Control}

The intersection from the previous section provides the target corner position. The algorithm converts this point to relative pan-tilt coordinate and dispatches a positional command to the motor control sub-system. As a whole, the proposed fixation algorithm forms a closed-loop visual feedback control structure.

\section{AdVAntage of THE LOG-POLAR FOR VOTE BASED ALGORITHMS}

Visual attention for vote-based algorithms is computationally simplified using the log-polar sensor. The highresolution fovea naturally gives rise to a higher pixel count when a corner is close to centre. This inherent bias fits well with the log-Hough transform process, which will find the line with more votes first (see Figure 7). Due to the logarithmic drop-off in towards the periphery, any line that is close to the centre will have a much greater pixel count for the same projected length. In the majority of cases, the two most dominant lines correspond to the lines that fall closest to the camera centre.

An important benefit of using the log-polar sensor with log-Hough line detection is the automatic bias for corners closer to fovea. This removes the need for explicit search through all detected corners, possibly a large number for cluttered scenes, to locate the corner closest to the centre. Each corner to be checked requires an explicit resolution of the position of the intersection of the component lines. Such a search would be necessary for a Cartesian sensor.

\section{IMPLEMENTATION}

Control calculation: We take the $\mathrm{n}$ lines with the highest vote count. The intersections of all pairs of these $n$ lines are solved using Equations (11 and 12). If multiple intersections exist, the algorithm may take the one with the smallest radial component $(r)$ as the estimated fixation point. This does not guarantee that a correct corner will always be found, but in most cases is sufficient to implement stable fixation. The results demonstrate stability in multi-corner scenes. 


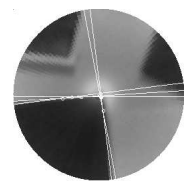

(a)

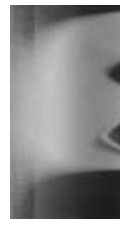

(b)

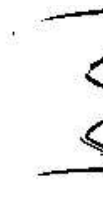

(c)

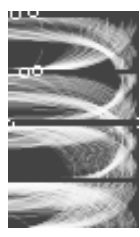

(d)
Fig. 8. False peaks around a true peak in Hough space causes detection of erroneous lines around edges. (a) Cartesian remapped image, (b) log-polar image, (c) edge map image and (d) Hough vote map.

Hough quantisation: The Hough space dimension of $60(\rho) \times 100(\theta)$ was chosen empirically to be a good compromise between computation expense and quantisation error. This Hough space dimension translates to a minimum resolvable line orientation $\left(3.6^{\circ}\right.$ in this case).

Hough peak finding and non-maximal suppression: Since the image space is discrete, quantisation errors cause a number of votes to fall into Hough bins that neighbour a peak. See [24], [13] for an analysis of the problem and some possible approaches to dealing with it.

We apply smoothing of Hough space to assist with this problem. However, edges of real world objects are often accompanied by shadows that appear as edges nearby. This problem is more important with fixation for real scenes. These two problems can combine to lead to multiple edges from a single object edge overwhelming other lower but significant local peaks, as shown in Figure 8. To overcome this problem, non-maximum suppression is applied around the current global peak. We clear a number of rows $(\theta)$ in both directions. The range of the non-maximal suppression could have been limited in $\rho$, but widely separated parallel lines will not have a corner, and so can be discarded. For the trials shown in this paper this was set to be around $14^{\circ}$. The setting that should be used depends on the thickness of lines in the operating environment, and the narrowness of corners that are required to be tracked. If edges were always thin this would not be necessary.

\section{RESUlTS AND ANALYSIS}

Extensive experiments have been conducted to evaluate the performance of the fixation algorithm. We conducted a series of trials with simple clean corners, such as Figure 1 , to evaluate the quantitative accuracy of corner localisation. We also tested different corner angles, more complex scenarios of multiple corners, and short corners to ensure stable fixation. Lastly, the real-time fixation performance was verified.

All trials were conducted with the Giotto camera and a custom pan tilt platform mounted on a Pioneer robot as shown in Figure 4. Current non-optimised performance with an Intel Pentium III 600MHz is around 20 frames per second, which is adequate for real-time fixation. Video of

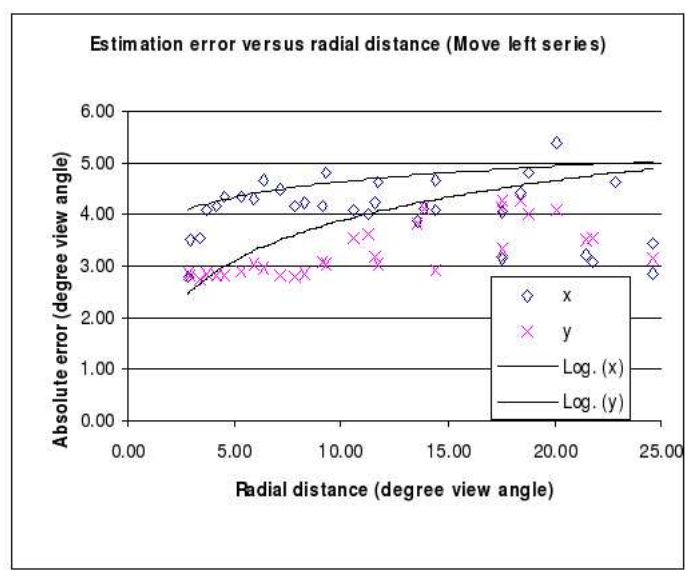

Fig. 9. Estimation errors versus radial distance (moving the camera left). Note that four outliers, three approximately $10^{\circ}$ and one $14.5^{\circ}$, are not shown for formatting clarity, but are included in the trend-line calculation.

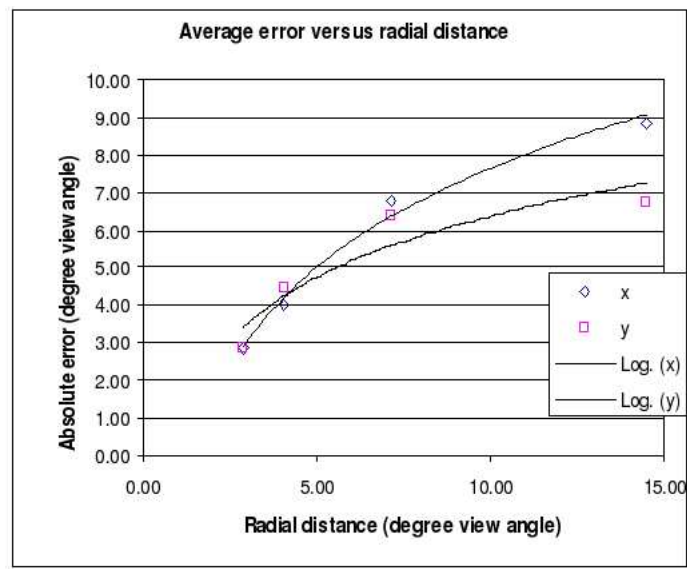

Fig. 10. Average estimation errors versus radial distance from the centre point.

the system demonstrating its closed-loop performance can also be seen at:

http://www.nicta.com.au/ nmb.

\section{A. Quantitative Verification}

In order to evaluate the precise error in estimation, we disabled the control system. The first trial examined stability with respect to distance from the image centre. A corner of 90 degrees, as shown in Figure 1, was moved to the left of the image across 39 trials. Figure 9 plots the position estimation error against radial distance. The error shown is the visual angular distance between the manually estimated corner point and the point estimated by the system. Visual angle from camera calibration gives a clearer result than the pixel count, which is non-linear. This reducing estimation error trend appears to be approximately logarithmic, which corresponds to camera sampling resolution as expected. High precision fixation at the fovea is an advantage of the log-polar sensor. Results for moving right were comparable. Thus, generally, an accurate estimate of corner position is obtained when a single clean corner is present. 


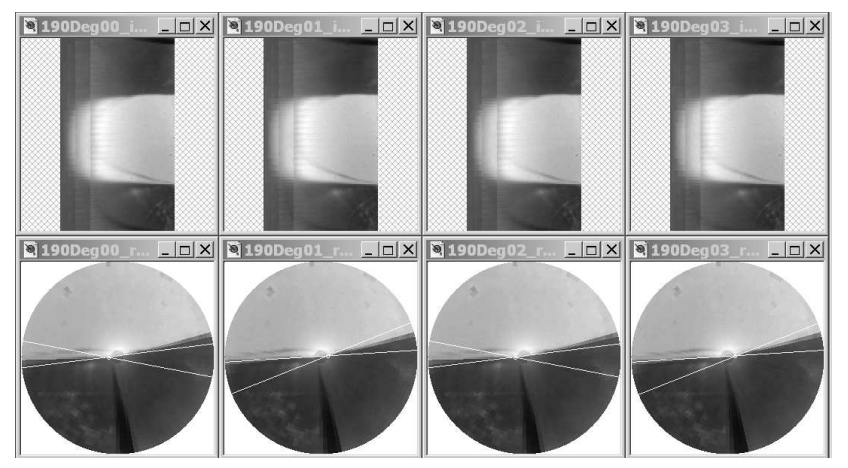

Fig. 11. Unstable fi xation for a $190^{\circ}$ corner target causes platform to oscillate around the centre.

To verify performance with different corner shapes and different directions of motion away from the image centre, we trailed 14 differently angled corners ranging from $15^{\circ}$ to $340^{\circ}$. Each was moved to four standard positions between the fovea and 15 degrees from it, at four $90^{\circ}$ intervals around the centre. The direction that the lines of the corner extended from the corner was also varied. Figure 10 shows the error at each position, averaged across all trials, demonstrating stability.

1) Corner angular dimension limit: Within the above trial, we tested nine different corner angles ranging from $15^{\circ}$ to $165^{\circ}$. For this range the error estimates were acceptable for fixation. Between $0^{\circ}$ to $15^{\circ}$ and $165^{\circ}$ to $180^{\circ}$ corners were not stably tracked, as shown in Figure 11. This corresponds to the minimum line orientation difference allowed by non-maximal suppression. Note that the range from $180^{\circ}$ to $360^{\circ}$ is symmetric to this and shows similar performance. Under low illumination, there was also instability close to minimum corner angle. This appeared to be due to slight edge blurring under low lighting.

\section{B. Special cases: multiple and short corners}

We trailed many cases of fixating a corner in multiple corner scenes. In the majority of cases where a significant corner is visible (see Figure 12,13), the intersection of the two major lines is correctly detected. However, in some ambiguous situations such as Figure 14, there are multiple corners close to the centre. Here the camera may move to either (whichever appears closer) but once it has moved, fixation will be stable.

Nevertheless, there exist degenerate cases, such as Figure 14 , where the centre-most line intersection is not a real corner. This can cause instability by saccading to the fictitious corner point. However, generally, this will not be a stable solution and will disappear given camera motion towards the non-existent corner, leading to stable fixation. With the camera being an active observer, prolonged erroneous states are rarely encountered. Figure 14(c) shows a case where incorrect fixation may be prolonged. This case could be corrected by additional processing to reject corners with no edge pixels close to the intersection.

For short corners, the algorithm maps all pixels of the
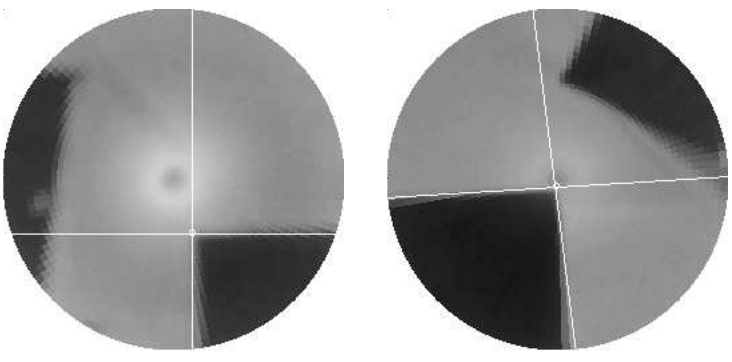

(a)

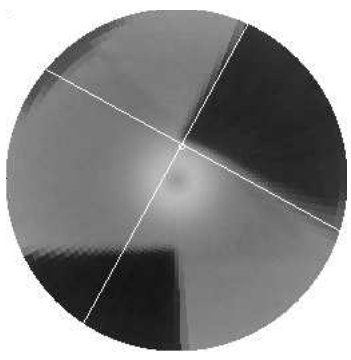

(c)

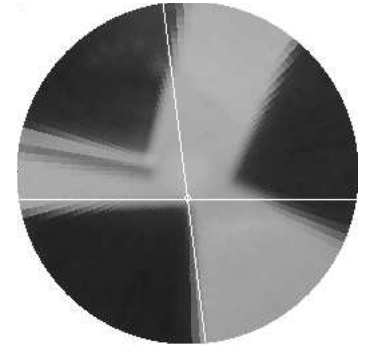

(d)
Fig. 12. Multi-corner scene - a dominant corner exists and is recognised correctly.

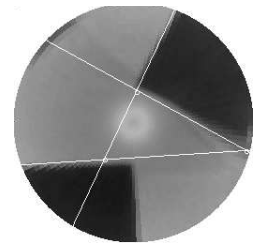

(a)

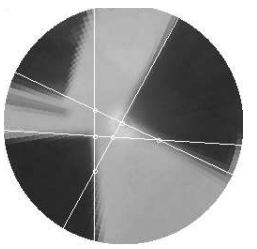

(b)

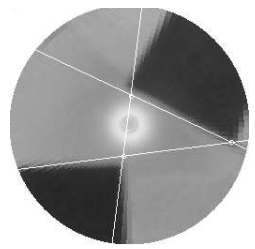

(c)
Fig. 13. Multi-corner scene - multi-intersection cases: (a), (b) are determinate cases due to proximity to the centre of one corner, whereas for (c) either corner may be selected.

edges that make up a corner, thus corners with adequate spatial extent are fixated stably (see Figure 15).

\section{Office scene}

In addition to these controlled cases, we also verified the system performing closed-loop with robot motion through many extended trials, including in two indoor scenes: the corner of a door window (Figure 16); and, the corner of a room (Figure 17). For both scenes fixation was run for several minutes and despite occasional poor estimates the fixation was stable. 


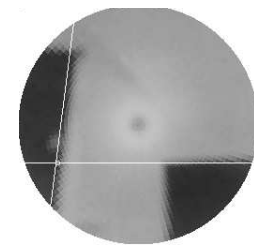

(a)

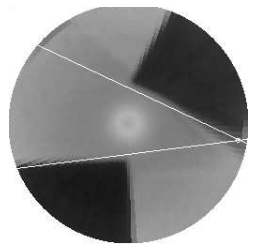

(b)

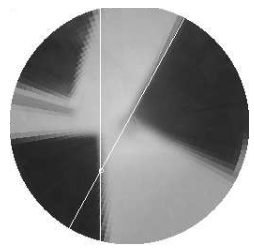

(c)
Fig. 14. Multi-corner scene - degenerate cases: (a) and (b) will disappear as soon as the camera moves, however for (c) the incorrect fi xation may be stable.

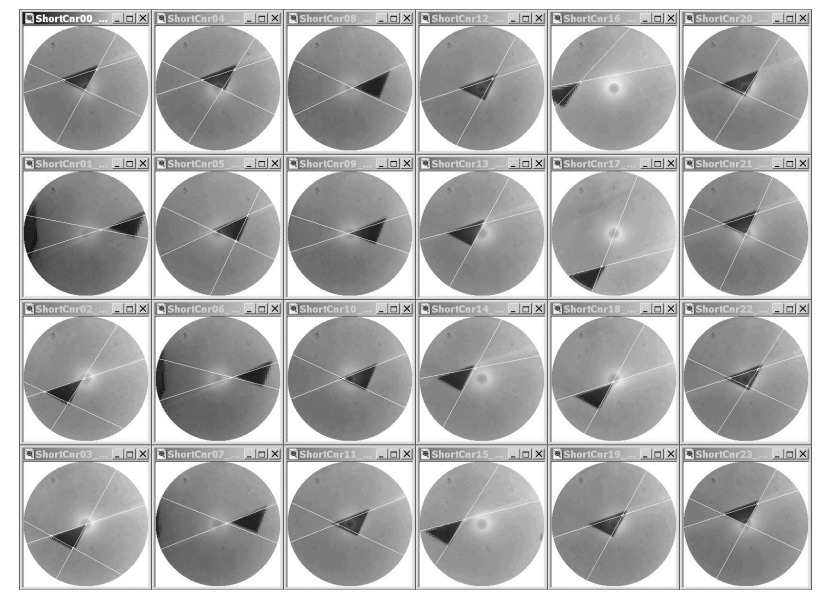

Fig. 15. Cartesian remapped images of short corners, demonstrating correct recognition of centre-most corners.

Figure 16 shows images taken at random intervals during this trial, displaying the estimated lines and corners. It can be seen that for the first sequence (see Figure 16), one fifth of the images show that the corner was not accurately detected. However, this did not prevent stable fixation. Figure 17 shows a difficult case for corner fixation where one edge is wide, due to self-shadowing under low illumination. Again, fixation was stable, even though a quarter of the cases verified were in error. For both trials the incorrectly detected corners are due to dominance of near parallel lines. This could be improved by tuning the algorithm, such as broadening non-maximal suppression. However, the absence of such tuning demonstrates robustness of the algorithm without parameter tuning. Fixation was stable despite many corner features being present and the fact that the algorithm is given no information to discriminate between them other than favouring central corners with spatial extent.

\section{CONCLUSION}

This paper presented a new log-polar corner fixation algorithm. Fixation is critical to the see the benefits of the logpolar sensor in robot vision. The paper also demonstrates the advantages of the log-polar sensor for fixation, that there
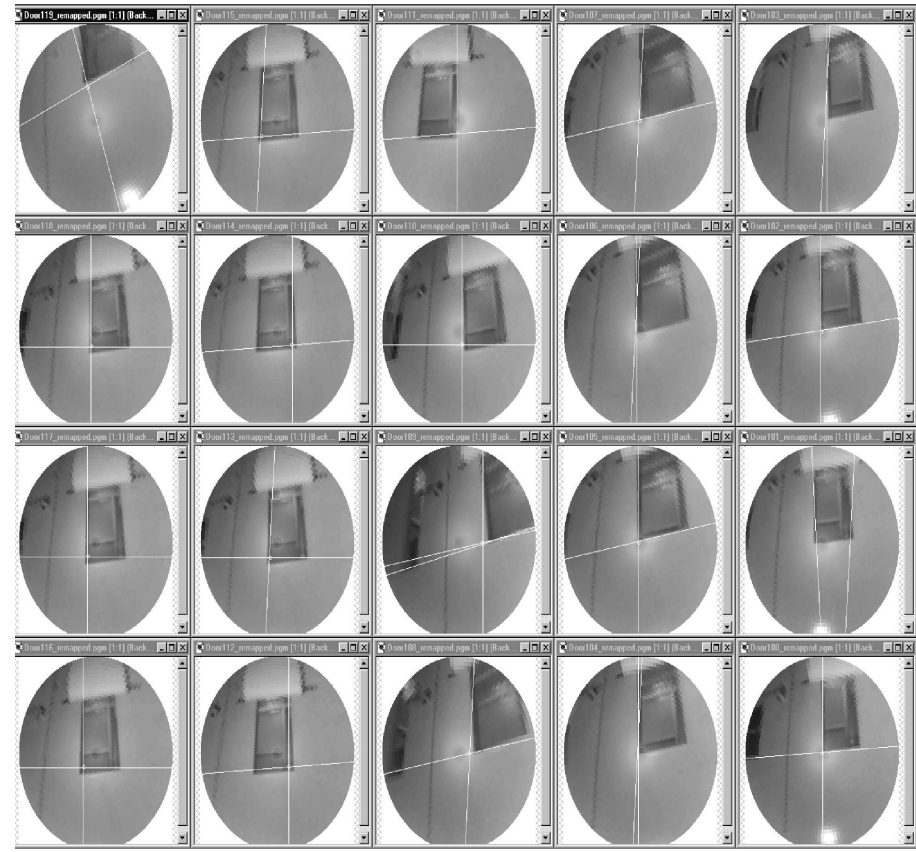

Fig. 16. Fixation on an indoor scene of a window.

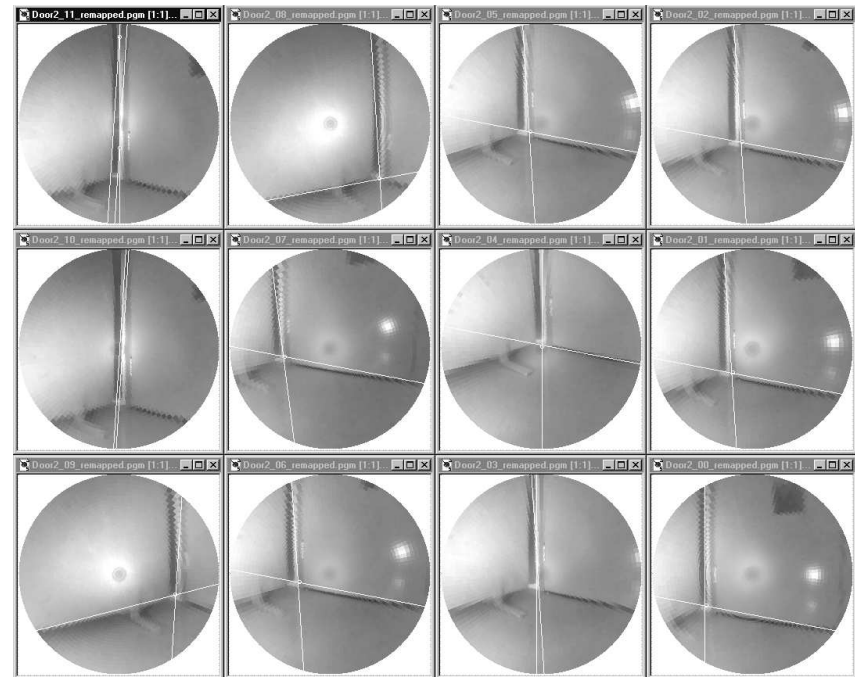

Fig. 17. Fixation on the corner of a door. Note the strong shadowing around the edge of the door.

is a reduced computational load for supporting high accuracy fixation at the fovea (reduced error in corner position estimation), while still maintaining a wide field of view. It also demonstrated advantages for vote-based algorithms in terms of the distance weighting of sensing elements aiding focus of attention. This advantage eliminated the need for explicit search through all corners in an image to locate the centre-most. In the course of developing the fixation algorithm, we fully derived the Hough transform voting equation in log-polar domain for real images. A full hardware and software closed-loop system was developed 
to implement the algorithm. Experimental verification has shown that the system is stable and has demonstrated realtime performance. This is an important result because it confirms the advantages of the log-polar sensor for this application. This paper has demonstrated that the log-polar sensor is highly suitable for fixation tasks where the focus of attention is at the high-resolution image centre.

\section{ACKNOWLEDGMENTS}

The authors would like to thank Professor Giulio Sandini for the use of the log-polar camera.

National ICT Australia is funded by the Australian Department of Communications, Information Technology and the Arts and the Australian Research Council through Backing Australia's ability and the ICT Centre of Excellence Program.

\section{REFERENCES}

[1] I Ahrns and H Neumann. Real-time monocular fi xation control using the log-polar transformation and a confi dence-based similarity measure. In International Conference on Pattern Recognition, pages 310-315, Brisbane Australia, Aug 1998.

[2] D H Ballard. Animate vision. Artificial Intelligence, 48(1):57-86, 1991.

[3] N M Barnes and G Sandini. Direction control for an active docking behaviour based on the rotational component of log-polar optic flow. In European Conference on Computer Vision 2000, volume 2, pages 167-181, 2000.

[4] A Bernardino and J Santos-Victor. Binocular tracking: integrating perception and control. IEEE Trans. on Robotics and Automation, 15(6), Dec. 1999

[5] M E Cleary and J D Crisman. Canonical targets for mobile robot control by deictic visual servoing. In IEEE Int. Conf. on Robotics and Automation, volume 4, pages 3093-8, 1996.

[6] K Deguchi and K Yonaiyama. Real-time 3d shape recognition of high speed moving object by fi xation point tracking with active camera. In Proc 2000 IEEE/RSJ Intl. Conf. on Intelligent Robots and Systems, volume 1, pages 596-601, 2000.

[7] R Deriche and G Giraudon. Accurate corner detection: An analytical study. In Int. Conf. on Conference on Computer Vision, pages 66-70, 1990.

[8] R O Duda and P E Hart. Pattern Classifi cation and Scene Analysis. Wiley, New York, 1973.

[9] N Gracias and J Santos-Victor. Underwater mosaicing and trajectory reconstruction using global alignement. In IEEE OCEANS 2001, Honolulu, USA, 2001.

[10] C Harris and M Stephens. A combined corner and edge detector. In Alvey Vision Conference, pages 147-151, 1988.

[11] P V C Hough. Method and means for recognizing complex patterns, December 1962. U.S. Patent, 3,069,654.

[12] Y B Jeon, B O Kam, S S Park, and S B Kim. Seam tracking and welding speed control of mobile robot for lattice type welding. In Proc. 2001 IEEE Intl. Symposium on Industrial Electronics, volume 2, pages 857-862, 2001.

[13] W Lam, L Lam, K Yuen, and D Leung. An analysis on quantising hough space. Pattern Recognition Letters, 15:1,127-1,135, 1994.

[14] S W Lee, B J You, and G D Hager. Object tracking using projective invariance. In IEEE Int. Conf. on Robotics and Automation, volume 2 pages 1589-1594, 1999.

[15] L Massone, G Sandini, and V Tagliasco. "form-invariant" topological mapping strategy for $2 \mathrm{~d}$ shape recognition. Computer Vision, Graphics, and Image Processing, 30(2):169-188, Apr. 1985.

[16] K Mikolajczk and C Schmid. Indexing based on scale invariant interest points. In Int. Conf. on Conference on Computer Vision, pages 525-531, 2001.

[17] K Mikolajczk and C Schmid. A performance evaluation of local descriptors. In IEEE Conf. on Conference on Computer Vision and Pattern Recognition, pages 257-263, Maddison, Wisconsin, USA, June 2003.
[18] N Oshiro, N Maru, A Nishikawa, and F Miyazaki. Binocular tracking using log polar mapping. In Proceedings of the 1996 IEEE/RSJ International Conference on Intelligent Robots and Systems.IROS1996, pages 791-8, 1996.

[19] P Questa and G Sandini. Time to contact computation with a spacevariant retina-line c-mos sensor. In Proceedings of the International Conference on Intelligent Robots and Systems, Osaka, Japan, 1996.

[20] E L Schwartz. A quantitive model of the functional architecture of human striate cortex with application to visual illustration and cortical texture analysis. Biological Cybernetics, 37:63-76, 1980.

[21] S Se, D Lowe, and J Little. Mobile robot localisation and mapping with uncertainty using scale-invariant visual landmarks. International Journal of Robotics Research, 21(8):735-758, Aug 2002.

[22] J Shi and C Tomasi. Good features to track. In IEEE Conf. on Computer Vision and Pattern Recognition, pages 593-600, 1994.

[23] M Tistarelli and G Sandini. On the advantages of polar and logpolar mapping for direct estimation of time-to-impact from optical flow. IEEE Trans. on Pattern Analysis and Machine Intelligence, 15(4):401-410, April 1993.

[24] T M vanVeen and F C A Groen. Discretization errors in the hough transform. Pattern Recognition, 14:137-145, 1981.

[25] H Wang, W L Goh, C S Chua, and C T Sim. Real-time object tracking. In Proc. of the 1995 21st Intl. Conf. on Industrial Electronics, Control, and Instrumentation, volume 2, pages 13661371, 1995.

[26] C F R Weiman. Polar exponential sensor arrays unify iconic and hough space representation. In Proceedings SPIE:Intelligent Robots and Computer Vision VIII:Algorithms and Techniques, pages 832842, 1989.

[27] C F R Weiman and G Chaikin. Logarithmic spiral grids for image processing and display. Computer Graphics and Image Processing, 11:197-226, 1979. 